Renata Marwege

\title{
Der Andengerichtshof
}

Das Rechtschutzsystem des Andenpaktes mit vergleichenden Bezügen zum Recht der Europäischen Gemeinschaft

Hamburger Studien zum Europäischen und Internationalen Recht, Band 4

Duncker \& Humblot, Berlin, 1995, 436 S., DM 138,--

$\mathrm{Zu}$ den Paradoxien lateinamerikanischer Integration gehört es, gemeinsam eine Richterbank besetzen und trotzdem Krieg gegeneinander führen zu können. Ecuador und Peru, Gründungsmitglieder mit Bolivien, Chile und Kolumbien des 1969 gebildeten Andenpaktes, ${ }^{1}$ als dessen gemeinsames Justizorgan seit 1983 der Andengerichtshof besteht, haben inzwischen zweimal die Klingen gekreuzt. Zwar konnten die im umstrittenen 340 qkm-Grenzgebiet der Cordillera del Condor züngelnden Flammen jedesmal rasch ausgetreten werden. Dies aber, wie zuletzt Anfang 1995, nur durch Bemühungen der Rio-Pakt-Garantiemächte Argentinien, Chile, USA unter Federführung Brasiliens und nicht etwa durch eine streitschlichtende, geschweige denn streitentscheidende Rolle des Andengerichtshofes. Der ist indes für dergleichen auch nicht konzipiert. Vielmehr sollte er, strukturell und kompetenziell dem Luxemburger EuGH nachgebildet, dem in den siebziger Jahren lahmenden andinen Integrationsprozeß neue Impulse geben.

Dieser neben Kommission und Rat ("Junta") erst 1988 zusammen mit dem schon 1979 geschaffenen Andenparlament zum Hauptorgan des Andenpaktes aufgewerteten Institution widmet sich die vorliegende Arbeit, eine bei Meinhard Hilf 1994 abgeschlossene Hamburger Dissertation. Durchforstet man ihr 359 Titel umfassendes Literaturverzeichnis, scheint der Andengerichtshof (AGH) trotz seiner Wahlverwandtschaft mit dem EuGH hierzulande so gut wie vergessen. Um so reizvoller die Herausforderung für die Autorin, die sich ihr ohne Wenn und Aber gewachsen gezeigt hat.

Die Arbeit ist plausibel gegliedert. Nach einer Einführung in Geschichte und Struktur des Andenpaktes - der inzwischen (1995/96) nach europäischem Vorbild zur "Andengemeinschaft" (Comunidad Andina) umgeformt wurde ${ }^{2}$ - wird im 2. Kapitel ein knappes Drittel des Buches allein auf die Entstehungsgeschichte des AGH-Gründungsvertrages vom 28. Mai 1979 (AGHV) verwendet. Dies rechtfertigt sich, wie die Autorin überzeugend darlegt, aus den mannigfachen souveränitätspolitisch bedingten Geburtswehen, eingedenk derer es dem Leser leichter fallen wird, auch aktuelle Schwachstellen lateinamerikanischer Integrationsperspektiven zu orten. Bei allem Detailreichtum dieses Kapitels überrascht nur eines: Nirgendwo erfährt man das Datum, an welchem dieser die ganze Arbeit tragende Vertrag nach seinem Art. 37 (Hinterlegung der letzten Ratifikationsurkunde beim Sekretariat der Kommission) in Kraft getreten ist. Das 3. Kapitel beschreibt Organstruktur und

1

2

Venezuela trat 1973 bei, Chile 1976 aus, Peru 1992 in den einstweiligen Passiv-Status.

Dazu im einzelnen Klaus Bodemer, Eine neue Chance für den Andenpakt?, NORD-SÜD aktuell 1996, S. $254 \mathrm{ff}$. 
Verfahrensbestimmungen des $\mathrm{AGH}$, ehe in den folgenden Kapiteln als dem eigentlichen Kern der Arbeit jeweils die drei Hauptverfahrensarten behandelt werden. Das 7. Kapitel geht kurz auf im AGHV nicht vorgesehene Verfahrensarten ein, gefolgt vom Schlußkapitel "Retrospektive und Perspektiven". Das Buch endet mit einem Verzeichnis aller verfügbaren AGH-Entscheidungen und einem Anhang, in dem sich der Andenpakt-Text in Alt- und (seit dem Protokoll von Quito 1988) Neufassung nebst Geschäftsordnungen von Junta und Kommission sowie der AGHV in spanisch und englisch nebst Statut und Geschäftsordnung des AGH finden.

Mit diesem kompletten Informationsspektrum ist die Arbeit für den deutschsprachigen Raum eine Pionierleistung, geschrieben übrigens in einem für juristische Verhältnisse erfreulich lesbaren Stil. Die Leitfrage, ob der AGH "... tatsächlich einen Beitrag zur Verwirklichung der Integration der Andenländer ... theoretisch leisten kann und in der Praxis leistet" (S. 22), wird kenntnisreich aufbereitet, das Luxemburger Pendant stets fest im Blick. So fest, daß die Darstellung der Verfahrensarten nicht der Reihenfolge des AGHV folgt, der mit der Nichtigkeitsklage beginnt, sondern dem EWG-Vertrag (EWGV), der diese Klageart erst an zweiter Stelle behandelt. Auch terminologisch löst sich die Autorin nicht vom EWGV: Die präzisen AGHV-Bezeichnungen "Acción de Incomplimiento" (Nichterfüllungsklage) und "Interpretación prejudicial" (Vorgreifliche Auslegung) bleiben zugunsten der deutschen Fehlübersetzungen "Vertragsverletzung" bzw. "Vorabentscheidung" (vgl. BGBl. 1957 II, S. 753 ff./870 ff.) auf der Strecke. Schade auch, daß die Entstehungsgeschichte des AGH so gut wie ausschließlich aus der Sekundärliteratur referiert wird. ${ }^{3}$ Mitunter beschwerlich auch die Lektüre des kapitelweise durchnumerierten Apparats: Immer wieder begegnet der Leser kompletten Quellenangaben, die ebenso noch einmal das Literaturverzeichnis füllen und diesem nur noch lexikalischen Nutzen belassen.

Alles in allem überzeugt die Arbeit jedoch. Ihr Resümee fällt differenziert aus: Strukturell und von den prozessualen Möglichkeiten her hält die Autorin den AGH für ohne weiteres in der Lage, "... den ihm zugedachten Beitrag zum andinen Integrationsprozeß - die Stärkung der andinen Rechtsordnung - zu leisten ..."; seine gleichwohl vergleichsweise geringe Inanspruchnahme führt sie darauf zurück, daß derzeit "... handelspolitische Maßnahmen der Mitgliedstaaten im Sinne einer regionalvölkerrechtlichen, administrativen Planung und politischen Zusammenarbeit ..." im Vordergrund stünden, was in diesem Integrationsstadium "... diplomatisch-administrative Verfahren ..." zur Konfliktlösung eher geeignet erscheinen lasse (S. 284 f.). Die Arbeit gewinnt schließlich dank ihrer erschöpfenden Verarbeitung des hauptsächlich (latein-)amerikanischen Schrifttums und der bisherigen Recht-

Hochinteressant und verständnisfördernd für etliche Detailregelungen im AGHV z.B. die Zusammenstellung der vielfach divergierenden Ländervoten aus den Einzelberatungen zum ursprünglichen Vertragsentwurf (Documento Auxiliar para el analisis de las observaciones formuladas por los paises miembros sobre la propuesta 43/Rev. 2 de la Junta, JUN/di 335 v. 8.9.1978) oder die Sachstandsberichte der Junta an die Kommission, z.B. COM/XXV/di 6 v. 23.10.1978. Unter anderem auch diese Quellen stellte seinerzeit die Junta dem Rezensenten für eine deutsche Übersetzung des AGHV bereitwillig zur Verfügung. 
sprechung des AGH über weite Strecken den Rang einer veritablen Kommentierung zum AGHV. Das läßt sie von erheblichem Interesse auch für den Praktiker sein, der sich im Zuge fortschreitender auch subregionaler lateinamerikanisch-europäischer Kooperation mit der Rechtsordnung im Andenraum vertraut machen möchte.

Karl-Andreas Hernekamp

\section{Guido Odendahl}

\section{Das Recht auf Entwicklung - The Right to Development}

Entstehungsgeschichte, systematische Stellung und Inhalt eines individuellen sowie kollektiven Menschenrechts und Grundprinzips der Völkerrechtsordnung Shaker Verlag, Aachen, 1997, 356 S., DM 89,--

Das Recht auf Entwicklung ist seit dem Vortrag Kéba M'Bayes vor dem International Institute of Human Rights in den 70er Jahren ${ }^{1}$ aus der Diskussion um das Verhältnis von Recht und Entwicklung ${ }^{2}$ nicht mehr fortzudenken. Auch vielfältige Kritik an der Herleitung eines solchen Rechts aus dem Völkervertrags- und Völkergewohnheitsrecht, an seinem Inhalt und seinen Adressaten, schließlich an seiner Durchsetzung haben es nicht vermocht, diesem Nomos seine zentrale Bedeutung für die Formulierung der rechtlichen Anforderungen an die Ordnung der internationalen Gemeinschaft streitig zu machen. Die Vielfalt von Diskussionsbeiträgen ${ }^{3}$ über einen geraumen Zeitraum hinweg macht es erforderlich, gelegentlich im Rahmen einer Bestandsanalyse eine erneute Standortbestimmung vorzunehmen. Die vorliegende Arbeit, eine von Professor Meinhard Schröder betreute Trierer Dissertation, wird ihrem Anspruch einer solchen Standortbestimmung und ihrem - doch recht umfänglichen - Untertitel weitgehend gerecht. Sie schildert eingehend die Entstehung des Rechts auf Entwicklung in seinen vielfältigen Ausprägungen mit seinen Ursprüngen im

Kéba M'Baye, Le droit au développement comme un droit de l'homme, Revue des droits de l'homme 1972, S. 502.

2 Vgl. nur Ernst Ulrich Petersmann, Völkerrecht und Entwicklungshilfe, VRÜ 5 (1972), S. 161, Rainer Tetzlaff, Die Forderungen der Entwicklungsländer nach einer ,neuen, Weltwirtschaftsordnung, VRÜ 9 (1976), S. 33, Hermann Weber, Der Anspruch auf Entwicklungshilfe und die Veränderungen des internationalen Wirtschaftsrechts, VRÜ 11 (1978), S. 5, Albert Bleckmann, Anspruch auf Entwicklungshilfe, VRÜ 12 (1979), S. 5,

3 Die Diskussion fand auch in dieser Zeitschrift ihren Niederschlag. Vgl. - außer den Nachweisen in Fn. 1 - vor allem Philip Kunig, Die "innere Dimension" des Rechts auf Entwicklung - Rechtspolitische Überlegungen zur Inpflichtnahme von Entwicklungsländern, VRÜ 19 (1986), S. 383 und Maria M. Kenig-Witkowska, The Paradigm of Development in International Law, Beiheft 14 zu VRÜ, Baden-Baden 1988, S. 78 ff., sowie die Buchrezensionen in VRÜ 12 (1979), S. 5; 20 (1987), 286; 21 (1988), S. 512 und 22 (1989), S. 519. 